\title{
Philosophiques
}

\section{Souci et connexion dans l'éthique de la politique générale}

\section{Victoria Davion}

Volume 22, numéro 1, printemps 1995

URI : https://id.erudit.org/iderudit/027308ar

DOI : https://doi.org/10.7202/027308ar

Aller au sommaire du numéro

Éditeur(s)

Société de philosophie du Québec

ISSN

0316-2923 (imprimé)

1492-1391 (numérique)

Découvrir la revue

Citer cet article

Davion, V. (1995). Souci et connexion dans l'éthique de la politique générale. Philosophiques, 22(1), 53-63. https://doi.org/10.7202/027308ar

\section{Résumé de l'article}

Ce texte porte sur l'élaboration des politiques publiques à partir d'une perspective conséquentialiste basée sur les probabilités. En utilisant l'exemple de la dissuasion par la menace nucléaire, je montre que ceux qui sont véritablement dévoués à la protection de l'environnement, ceux qui se soucient véritablement du problème de l'environnement devraient refuser le conséquentialisme basé sur les probabilités sans le principe de la reconsideration, un principe que nous proposons ici pour la première fois, le principe de la reconsideration doit être adopté parce que, sans lui, le conséquentialisme basé sur les probabilités pourrait justifier qu'on poursuive l'application de politiques même lorsque celles-ci se sont révélées inadéquates, et même lorsqu'elles minent elles-mêmes leurs chances de succès. On peut ainsi conclure que le principe de la reconsidération est nécessaire pour assurer le lien entre les politiques justificatrices et leur succès. 
Philosophiques, vol. XXII, NUMÉro 1, Printemps 1995, P. 53-63

\title{
SOLCI ET CONNEXION DANS L'ÉEHIOUE DE LA POLITIQUE GENÉRALF*
}

\author{
par \\ Victoria Davion
}

\begin{abstract}
RÉSUME : Ce texte porte sur l'élaboration des politiques publiques à partir d'une perspective conséquentialiste basée sur les probabilités. En utilisant lexemple de la dissuasion par la menace nucléaire, je montre que ceux qui sont véritablement dévoués à la protection de l'environnement, ceux qui se soucient véritablement du probleme de l'environnement devraient refuser le conséquentialisme basé sur les probabilités sans le principe de la reconsidération, un principe que nous proposons ici pour la première fois. Le principe de la reconsidération doit être adopté parce que, sans lui, le conséquentialisme basé sur les probabilités pourrait justifier qu'on poursuive l'application de politiques même lorsque celles-ci se sont révélées inadéquates, et même lorsqu'elles minent elles-mêmes leurs chances de succés. On peut ainsi conclure que le principe de la reconsidération est nécessaire pour assurer le lien entre les politiques justificatrices et leur succès.
\end{abstract}

ABSTRACT: This paper examines the making of public policy from a probability-based consequentialist perspective. Using nuclear deterrence policies as an example, I show that those truly committed environmental protection, those who truly care about the environment should not accept probability-based consequentialism without the reconsideration principle, a principle introduced in this paper. The reconsideration principle must be accepted, because without it, probability-based consequentialism can justify continuing to act on policies even when they have failed, and even when they undermine their original goal altogether. Thus, we conclude that the reconsideration principle is needed to insure connection between the justification policies and their success.

Cet article examine l'élaboration de la politique générale dans une perspective conséquentialiste basée sur la probabilité. En prenant pour

* Je remercie les éditeurs de Philosophiques de leurs utiles suggestions. Je voudrais remercier aussi le Humanities Center de l'Université de Géorgie d'avoir mis à ma disposition les fonds nécessaires à ce projet. 
exemple la politique de dissuasion nucléaire, je montrerai que ceux qui s'intéressent vraiment à la protection de l'environnement, ceux qui se soucient vraiment de l'environnement ne devraient pas accepter le conséquentialisme basé sur la probabilité sans le principe de reconsidération, principe présenté dans cet article. Le principe de reconsidération doit être accepté, car, sans lui, le conséquentialisme basé sur la probabilité peut justifier la poursuite d'une politique même lorsqu'elle a dejjà échoué, et même lorsqu'elle mine complètement son but originel. Je soutiendrai donc que le principe de reconsidération est nécessaire pour garantir la connexion entre la justification de la politique et sa réussite.

Le principe de reconsidération formule que:

Pour toute politique $\mathbf{P}$ et tout temps $\mathrm{T}$,

la justification conséquentialiste de $\mathrm{P}$ à $\mathrm{T}$

exige que $P$ rende probable une augmentation

d'utilité à $\mathrm{T}$ ou dans l'avenir.

Il semblerait que le conséquentialisme basê sur la probabilité implique le principe de reconsidération, puisque ce principe incarne l'esprit même du conséquentialisme qui exige que le but d'une politique soit de générer des consẽquences positives. Toutefois, dans le contexte des débats sur la dissuasion nucléaire, David Gauthier a suggéré une justification conséquentialiste des politiques de dissuasion nucléaire qui implique le rejet du principe de reconsidération ${ }^{\mathrm{I}}$. Dans ce qui suit, je présenterai la position de Gauthier, et je montrerai pourquoi les politiciens qui se soucient vraiment de la protection de l'environnement, et qui veulent maintenir une connexion entre la justification de leur politique et l'avancement des objectifs que cette politique avait pour mission de faire avancer, ne devraient pas accepter cette position. Ainsi, je montrerai que des politiciens soucieux de bien faire doivent rejeter l'approche de Gauthier parce qu'elle nie implicitement le principe de reconsidération ${ }^{2}$.

Gauthier examine la situation suivante. Une nation préfère qu'il n'y ait aucune frappe nucléaire plutôt qu'une seule frappe, et après cela, elle préfère le plus petit nombre possible de frappes au total. Le résultat que cette nation désire le plus est une dissuasion réussie, tandis que le résultat qu'elle désire le

I. David Gauthier, « Deterrence, Maximization, and Rationality », Ethics 94, avril rg84, p. $474-495$.

2. Pour des discussions d'autres aspects de la justification des politiques de dissuasion nucleaaire, voir Anthony Kenny, The Logic of Deterrence, Chicago, The University of Chicago Press, ig85; Nuclear Deterrence préparé par Russell Hardin, John J. Mearsheimer, Gerald Dworkin, et Robert E. Goodin, Chicago, The University of Chicago Press, 1985; et Nuclear Deterrence and the Future of Humanite, préparé par Avener Cohen et Steven Le, New Jersey, Rowman et Allanheld, rg86. 
moins est de devoir déclencher sa propre frappe ${ }^{3}$. Les politiciens de cette nation pensent que la meilleure façon de garantir la dissuasion est de menacer sincèrement de représailles sous forme de frappe nucléaire ${ }^{4}$. Dans une telle situation, la tentative d'obtenir la dissuasion entraine la possibilité que cette nation crẻe le résultat qu'elle désire le moins, c'est-à-dire les représailles 5 .

Apparemment, il ne peut pas être rationnel pour une nation dont nous venons de voir les priorités, de mettre à exécution ses menaces de représailles. Une fois que la dissuasion a échoué, la nation n'aurait aucune raison d'user de représailles, et, en fait, aurait des raisons de ne pas le faire, puisque l'absence

3. Ce raisonnement présuppose que les représailles sont mauvaises. On peut penser cela pour plusieurs raisons. D’abord, si les frappes visent les populations civiles, cela constitue une violation du principe central de la théorie d'une guerre juste, le principe de discrimination. Ce principe formule que les attaques intentionnelles directes sur des non-combattants et sur des cibles non militaires sont interdites. Si la frappe vise les cibles militaires, mais a pour conséquence de nuire à de nombreux civils, elle peut être une violation du principe de proportionnalité des moyens militaires qui formule que (r) tout moyen militaire doit être proportionné à une fin militaire discrète, et que (2) les moyens militaires doivent être proportionnés à l'objet de la guerre, c'est-à-dire à une juste cause. Pour de plus amples discussions de ces principes, voir William V. O'Brien, The Conduct of Just and Limited Wars, Praeger Publishers, 1981; Michael Walzer, Just and Unjust Wars, New York, Basic Books, 1977; et Thomas Nagel, « War and Massacre », dans Mortal Questions, Cambridge, Cambridge University Press, 1979. p. 53-72.

4. On pourrait soutenir que, puisque des menaces mensongères peuvent être aussi efficaces que des menaces sincères, cette supposition est injustifiée. La dissuasion dépend de la crédibilité et non de la sincérité. Cependant, de nombreux théoriciens ont affirmé que le bluff n'est pas une option possible pour les théoriciens de la dissuasion nucléaire, à cause de la possibilité que d'autres nations apprennent la vérité, et des problèmes que cela entraînerait de dissimuler un tel secret aux citoyens de la nation qui bluffe. Je ne présume pas qu'il n'y ait aucune situation dans laquelle le bluff est une option, mais qu'il pourrait y avoir des situations dans lesquelles, à cause des problèmes qu'implique le bluff, les politiciens pourraient raisonnablement penser qu'il est trop dangereux. Pour une discussion plus poussée sur ce sujet, voir Anthony Kenny, « Counter Force and Counter Value » et « The Logic of Nuclear Deterrence », tous deux dansThe Ivory Tower: Essays in Philosophy and Public Policy. Londres, Basil Blackwell Press, I985; également les évêques catholiques américains, « On the Use of Nuclear Weapons and Nuclear Deterrence », dans James P. Sterba (dir.), The Ethics of War and Nuclear Deterrence, Californie, Wadsworth Publishing Company, 1985, p. 139-148.

5. C'est la nature paradoxale des stratégies de dissuasion nucléaire basées sur des menaces sincères d'entraîner des résultats extrêmement négatifs. Pour une analyse approfondie de ces paradoxes, voir Gregory Kavka, Moral Paradoxes of Nuclear Deterrence, New York, Cambridge University Press, 1987, en particulier les deux premiers chapitres. 
de représailles réduirait le nombre total de frappes. Il semble qu'une telle nation soit incapable de poursuivre la meilleure politique de dissuasion, puisqu'elle sera incapable de mettre ses menaces à exécution, et par conséquent, sera incapable de menacer sincèrement de dissuasion.

Gauthier soutient qu'une nation dont les priorités seraient celles que nous avons indiquees, pourrait justifier des représailles, dans certaines circonstances, si la dissuasion échouait. Il soutient que, pour évaluer une politique de dissuasion, les politiciens ne devraient pas considérer seulement la question de savoir si des représailles réelles seraient d'une utilité maximum. En faisant cela, ils négligeraient le fait qu'une intention sincère d'user de représailles a en soi des conséquences sur l'augmentation de l'utilité. A cause de cela, il est possible de justifier la politique de dissuasion en prenant pour base l'utilité prévue en formant l'intention. Selon Gauthier :

Le raisonnement en faveur de l'irrationalité de la dissuasion ne tient compte que du coût de l'échec de la dissuasion. Parce que ces coûts existent, il rejette la politique de dissuasion. Au contraire, mon raisonnement compare les coûts de l'échec de la dissuasion aux bénéfices de la réussite de la dissuasion, en tenant compte de la probabilité de chaque résultat, pour évaluer la rationalité de former l'intention conditionnelle et non maximisante qui est le fondement de la politique de dissuasion. Je prétends que, s'il est rationnel de former l'intention conditionnelle et dissuasive. alors, si la dissuasion échoue et si la condition se réalise, il est rationnel de passer à l'action. Le coût qu'entraîne la mise en œuvre de l'intention dissuasive est pris en compte, avec l'estimation probable adéquate, dans la question de savoir s'il est rationnel de former l'intention. Mais une fois la décision prise, le coût de la mise en œuvre de l'intention n'est plus pris en compte dans la question de savoir si, au cas où la dissuasion échouerait, il est rationnel de passer à l'action. Passer à l'action est une partie intégrante de la politique de dissuasion, et si l'utilité prévue est maximisée en formant l'intention conditionnelle et maximisante, il s'ensuit que la dissuasion est une politique rationnelle ${ }^{6}$.

Selon Gauthier, on peut justifier le coût de former l'intention sincère, y compris la possibilité que l'on doive passer à l'action, si l'utilité prévue en formant l'intention est assez élevée. Par conséquent, s'il existe une très forte probabilité que former l'intention dissuasive réussira à empêcher un agresseur potentiel d'attaquer, et que le risque que l'on doive vraiment passer à l'action est assez faible, on peut justifier l'intention. Cela signifie qu'il est justifié de la mettre en œuvre, parce qu'il ne peut pas être rationnel de former une intention qu'il est irrationnel de mettre en œuvre. Si l'on reconnaît l'irrationalité de passer à l'action, alors on sera incapable d'en avoir sincerement l'intention. On saura à l'avance qu'on serait incapable de le faire, et cela empêchera la possibilité d'en avoir sincèrement l'intention.

6. David Gauthier, loc, cit., p. 486. 
L'explication de Gauthier pour justifier des représailles si la dissuasion échouait, l'amène à affirmer que, même si des représailles ne peuvent apporter que des conséquences indésirables, et même si l'on peut encore créer un résultat plus conforme à ses désirs en s'abstenant de représailles, il est moralement permis d'user de représailles. Selon Gauthier :

La dissuasion qui èchoue à dissuader et qui doit alors mettre à exécution ses menaces pour concrétiser son intention conditionnelle, n'est pas maximisante du tout. Elle maintient sa position non pas pour montrer l'exemple aux autres, ni pour améliorer sa perspective future, ni rien de tel, mais simplement parce que l'utilité ou la récompense prévue par cette politique manquée dépendait de sa volonté de maintenir sa position?

Le partisan de la dissuasion, selon Gauthier, fait une chose rationnelle en continuant l'intention plutôt qu'en changeant de plan, pour la simple raison que la politique manquée dépendait de la sincérité de l'intention pour être efficace. On a déjà rendu compte du coût de l'échec de la politique, on a déjà adopté la politique, et donc, on a déjà déterminé que tous les aspects de la politique sont permis. Gauthier justifie la continuation de la mise en œuvre d'une politique manquée en se tournant vers le passé plutôt que vers l'avenir. Il soutient que l'on devrait rester fidèle à sa politique et créer un résultat extrêmement négatif parce qu'au départ, il fallait accepter de faire cela pour que la politique ait une chance de réussir. Sa justification ne tient pas du tout compte des conséquences futures à partir du moment où la dissuasion échoue.

Le propos de Gauthier implique clairement le rejet du principe de reconsidération. Rappelons que le principe de reconsidération formule que, pour toute politique $P$ et tout temps $T$, la justification conséquentialiste de $\mathbf{P}$ à T exige que P rende probable une augmentation d'utilité à T ou dans l'avenir. Il fait espérer des conséquences souhaitables, ou du moins, les conséquences les plus souhaitables possibles dans des circonstances particulières, ce qui est une condition nécessaire pour poursuivre l'exécution de toute politique d'intérêt public. En ce qui concerne la politique de dissuasion nucléaire, le principe de reconsidération nous dit que, à partir du moment où la dissuasion échoue, on ne peut pas justifier la poursuite de la mise en œuvre de la politique manquée. Une réevaluation à $\mathrm{T}$ (au moment où la politique échoue) montre qu'on ne peut prévoir aucune conséquence souhaitable à $\mathrm{T}$ ou dans l'avenir si l'on met en œuvre la politique en usant de représailles. Comme le déclare Gauthier, ses raisons de continuer la politique n'ont rien à voir avec les conséquences futures.

Gauthier base le problème de la justification sur la question de savoir s'il est plus rationnel de planifier ses actions à l'avance ou de toujours évaluer sa 
situation au moment présent. Il soutient que planifier à l'avance permet de soumettre de plus grandes parties de sa vie à un examen rationnel. Il déclare:

L'acteur entièrement rationnel n'est pas celui qui évalue ses actions à partir du moment présent, mais plutôt celui qui soumet les plus grands, et non les plus petits, segments de son activité à un examen rationnel primaire, allant des principes aux performances, l'évaluation de celles-ci étant contrôlée par l'évaluation de ceux-là ${ }^{8}$.

Donc, Gauthier soutient que le problème central de la justification nécessite la rationalité de coordonner ses actions individuelles pour obtenir un effet maximisant à long terme, par opposition au fait de considérer les actions individuellement sans coordination à long terme. Pour une politique d'intérêt public, cela revient à examiner l'utilité d'une politique d'ensemble plutôt que l'utilité des actions individuelles qui forment cette politique.

Si l'on base le problème sur la question de la coordination des actions individuelles à l'intérieur d'une politique, il apparaît que Gauthier a certainement raison. Nous devons coordonner les actions individuelles ou nous ne pouvons espérer que le chaos. Cependant, je pense que Gauthier présente le problème sous un faux jour. La question réelle n'est pas de savoir s'il faut calculer l'utilité prévue des actions individuelles ou s'il faut calculer l'utilité prévue d'une politique d'ensemble. Le vrai problème concerne la réévaluation d'une politique. Cette réévaluation peut tenir compte de politiques d'ensemble plutôt que d'actions individuelles, et, par conséquent, n'empêche pas forcément de considérer la vision globale dans la coordination des actions individuelles comme parties d'une politique.

Toutes les discussions des mérites de l'évaluation d'une politique d'ensemble n'impliquent pas le rejet du principe de reconsidération. Dans son célèbre article «Two Concepts of Rules ». John Rawls explique comment les utilitaristes peuvent justifier certaines actions individuelles qui en ellesmêmes ne maximisent pas l'utilité, et sa justification n'implique pas le rejet du principe de reconsidération ${ }^{9}$. Pour montrer cela, il distingue entre deux conceptions des règles, le concept de pratique et le concept de résumé. Selon le concept de résumé, une règle indique quelle ligne d'action entraînera vraisemblablement une utilité maximale. Elle résume les applications passées du principe d'utilité dans des situations semblables. Selon le concept de résumé des règles, chaque fois qu'une réponse donnée en appliquant la règle entre en conflit avec la réponse donnée par une application directe du principe d'utilité dans une situation donnée, on doit suivre le principe lui-même. La règle est un raccourci auquel on doit passer outre dans des situations où elle entre en

8. Ibid., p. 488.

9. John Rawls, « Two Concepts of Rules », The Philosophical Review, 64, 1965. p. 3-32. 
conflit avec le principe d'utilité. Ceux qui se conforment au concept de résumé des règles essaient toujours d'atteindre l'utilité maximum à travers chaque action individuelle.

Par contraste, selon le principe de pratique des règles, on devrait appliquer le principe d'utilité au niveau d'une politique plutôt qu'au niveau des actions individuelles. Il sera permis à certaines actions individuelles de ne pas atteindre l'utilité maximum en elles-mêmes, pourvu qu'elles participent à une politique qui atteint l'utilité maximum.

Rawls démontre la différence entre ces deux visions du statut des règles en les appliquant au cas de la punition des innocents. Une objection que l'on fait généralement à l'utilitarisme est qu'il justifie la punition d'un innocent dans certaines situations où l'on ne peut pas trouver le coupable. Punir un innocent tout en déclarant qu'il est coupable pourrait être la meilleure façon dont nous disposons de dissuader d'autres personnes de commettre des crimes semblables, ou simplement une façon d'apaiser les gens s'ils croient que le coupable a vraiment èté puni ${ }^{\text {IO }}$.

Cette tromperie peut se justifier par le concept de résumé des règles, parce qu'elle est peut-être la meilleure façon d'atteindre l'utilité maximum. Pourtant, elle ne peut pas se justifier par le concept de pratique. Dans ce cas, la question pertinente est de savoir si une telle politique permettrait d'atteindre en général l'utilité maximum. Le principe s'applique seulement au niveau des décisions politiques. Il est évident qu'une telle politique ne serait pas une bonne chose. Tôt ou tard, les gens découvriraient le pot aux roses et perdraient toute confiance dans le système judiciaire, sans compter qu'ils penseraient pouvoir, eux aussi, être punis même s'ils n'ont rien fait de mal. Les gens ne pourraient pas se fier les uns aux autres.

Gauthier utilise une stratégie semblable en distinguant entre considérer une action individuellement et la considérer comme faisant partie d'une politique. On ne peut pas justifier les représailles comme acte individuel parce qu'elles diminuent l'utilité. Cependant, lorsqu'on les considère comme faisant partie d'une politique qui pourrait empêcher un désastre nucleaire, on peut les justifier quand les probabilités sont en faveur du succès de la dissuasion. L'exemple de la punition montre que Gauthier a raison. Pourtant, il est important de faire remarquer pourquoi. S'il est nécessaire de considérer ses décisions comme faisant partie d'une politique, c'est pour garantir les meilleurs

Io. Cet exemple a été introduit dans la bibliographie sur le sujet par H.J. McCloskey dans «A Note on Utilitarian Punishment », Mind 72, 1963, p. 599. Pour une discussion intéressante de l'utilitarisme de la règle contre l'utilitarisme de l'acte, voir J. C. C. Smart, «An Outline of a System of Utilitarian Ethics », dans Utilitarianism : For and Against, Cambridge, Cambridge University Press, 1973. 
résultats possibles. Alors qu'il n'est peut-être pas vrai que punir un innocent une fois entrainerait des conséquences désatreuses, il est vrai qu'une politique impliquant la punition des innocents en entraînerait vraisemblablement. Toutes les raisons données pour justifier de considerer ses actions comme faisant partie d'une politique plutôt que comme de simples actions individuelles sont liées à l'effet de ces actions dans l'avenir.

La justification de Rawls pour punir seulement l'innocent repose sur le fait que ceci va vraisemblablement produire de bons résultats. Contrairement au raisonnement de Gauthier, elle implique que si, à n'importe quel moment, on détermine que la politique d'ensemble ne marche pas, on devrait la changer. Ainsi, non seulement est-elle compatible avec le principe de reconsidération, mais elle implique la vérité de ce principe. La question-clé n'est pas de savoir s'il faut s'occuper des politiques plutôt que des actions individuelles, mais de savoir s'il faut regarder vers l'avenir ou vers le passé pour décider de la poursuite d'une certaine politique. Le principe de reconsidération affirme que l'on doit regarder vers l'avenir plutôt que vers le passé lorsqu'on évalue une politique. Il soutient que les décisions politiques doivent être tournées vers le futur.

Bien sûr, si l'on croit que, par des représailles, on peut encore, de quelque manière, obtenir le meilleur résultat possible, en limitant de futures attaques par exemple, alors on peut justifier des représailles. Pourtant, une fois encore, c'est parce que l'on espère un bien futur de cette politique. La décision d'user de représailles dans ces circonstances serait compatible avec le principe de reconsidération.

Je vais maintenant me pencher sur la question de savoir si les gens qui se sentent vraiment concernés par l'environnement peuvent accepter la position de Gauthier. Comme je l'ai déclaré dans l'introduction, je vais soutenir que non. Mon raisonnement sera centré sur la question de savoir quel sens donner à cette priorité accordée à la protection de l'environnement. La position de Gauthier implique qu'il est rationnel qu'une nation dont la priorité de ne donner lieu à aucune frappe est basée sur le désir de protéger l'environnement, se lance dans des représailles même quand aucun bien futur ne peut en résulter, et même si les représailles sont sa demière priorité. C'est rationnel parce que la volonté d'user de représailles était nécessaire pour que la politique ait une chance de réussir.

L'objectif de la nation ne change pas une fois que la politique a échoué. Quelles raisons réelles Gauthier peut-il apporter aux représailles ? Les politiciens ne seraient pas touchés par le fait qu'une intention sincère était nécessaire pour que la politique réussisse. En fait, cela n'a rien à voir avec leur objectif présent, qui est de limiter la dévastation nucléaire totale de l'environnement. Ils devraient changer leur objectif pour continuer d'agir 
selon la politique par des représailles. Leur nouvel objectif serait de devoir faire tout ce que leur politique dit qu'ils feraient, même si cela provoque un désastre.

Il est irrationnel que ceux qui ont pour objectif sincère d'empêcher un désastre de l'environnement n'incorporent pas le principe de reconsidération. S'ils ne le faisaient pas, ils iraient à l'encontre de leur objectif d'ensemble, celui que leur politique de dissuasion devait aider à atteindre, qui est d'empêcher ou de limiter la devastation de l'environnement. L'amour de l'environnement amènera les gens à baser leurs actions sur les effets de celles-ci sur l'environnement. et non sur ce qu'il faut faire pour qu'une politique manquée ait eu une chance de réussir.

L'élément d'incertitude doit toujours être un facteur pour le conséquentialisme basé sur la probabilité. En élaborant une politique générale, notre but d'ensemble doit être de produire le meilleur avenir possible. C'est l'idée clé que fournit le conséquentialisme. Pourtant, si nous acceptons cette idée, alors nous devons accepter une constante réévaluation de la politique. Chaque fois qu'une politique va mener au désastre, elle doit être changée.

En rejetant le principe de reconsidération, on perd beaucoup, sinon tout, de ce qui est séduisant dans le conséquentialisme. Le conséquentialisme nous permet de nous occuper de notre bien-ètre présent et à venir. Les adeptes du conséquentialisme déclarent souvent que les déontologues vénèrent des principes plutôt que le développement du bien concret dans le monde. Pourtant, un conséquentialisme sans le principe de reconsidération s'expose à la mème accusation, et ne s'oriente vraiment pas vers les conséquences. Plutôt que de nous faire vénérer des principes abstraits, le conséquentialisme privé du principe de reconsidération nous fait vénérer des décisions passées, même lorsque cela provoque des conséquences désastreuses. On perd l'esprit du conséquentialisme qui implique la production des meilleurs resultats possibles.

Bien que cette discussion soit centrée sur la politique de dissuasion nucléaire, elle a des répercussions sur la politique de dissuasion en général. chaque fois qu'une politique de dissuasion est basée sur une menace de faire quelque chose qui diminue l'utilité au cas où la politique échouerait. l'utilisation du conséquentialisme basé sur la probabilité sans le principe de reconsidération menace de détruire la connexion entre les raisons de continuer la mise en ceuvre de cette politique et la promotion de son but originel, pour aller vers quelque chose de très différent. Une fois que ce revirement justificatif est accompli, on a des raisons de rester fidèle à la politique quels que soient les résultats. La question-clé n'est pas que la dissuasion nucléaire soit en 
cause, mais que la dissuasion soit en cause, et que l'effet de dissuasion soit basé sur une menace de faire quelque chose qui diminue l'utilité à longue échéance.

Étant donné la tendance actuelle dans le développement des armes mortelles, on peut s'attendre très prochainement à des stratégies de dissuasion concernant les armes chimiques. Si ces politiques impliquent une menace sincère de faire quelque chose qui est désastreux en soi, les mêmes problèmes vont se poser à propos de ces politiques. En outre, cette discussion a des répercussions sur des politiques qui n'ont rien à voir avec la dissuasion. Elle pose la question plus générale du moment opportun de reconsidérer une politique. Gauthier suggère qu'il y a des moments où il est mauvais de reconsidérer une politique. Il pense que la démarche rationnelle exige que, pour certains types de politiques, la question de savoir s'il faut les appliquer ne peut se poser qu'une seule fois. Cela nous permet de soumettre de plus grandes portions de notre vie à un examen rationnel. Sa position générale est qu'on doit avoir la vision la plus globale possible lorsqu'on fait des projets pour l'avenir. Mais quelle est la vision globale ? C'est sur ce point que nous ne sommes pas d'accord. Gauthier commence par adopter une démarche qui envisage les moyens en vue d'une fin, puis procède à un revirement par lequel les moyens deviennent le centre d'attention plutôt que la fin. Selon son analyse, les gens qui commencent par créer une politique d'intérêt public pour empêcher quelque chose peuvent finir par causer la chose même qu'ils avaient l'intention d'empêcher. Je pense que cela revient à perdre de vue la vision globale, l'importance du but originel. Du point de vue des écologistes radicaux, être soi-même la cause du désastre qu'on veut empêcher, seulement parce que c'est ce qu'il aurait fallu faire pour que la politique réussisse, est de la folie. Si l'on se soucie vraiment de l'environnement, on abandonnera la politique manquée et l'on continuera à s'efforcer de préserver l'environnement. On ne laissera pas la politique déterminer le but, mais on insistera pour que le but détermine la politique. Par conséquent, on voudra absolument que les raisons de continuer à mettre en œuvre une politique aient une connexion particulière avec le but que cette politique doit permettre d'atteindre, une connexion qui serve à le faire avancer. On n'admettra pas la sorte de déconnexion radicale entre les raisons d'appliquer une politique et les raisons de continuer à l'appliquer, suggérée par Gauthier.

De façon plus générale, je pense que la théorie de Gauthier n'est pas pratique, puisqu'elle ne peut pas être appliquée par ceux qui se soucient vraiment d'atteindre certains buts dans le monde réel. Ceux qui se soucient uraiment d'atteindre certains buts n'agiront pas d'une façon qui garantisse virtuellement que ces buts ne seront pas atteints. La position de Gauthier est peut-être cohérente, mais elle est si peu réaliste qu'elle en devient inutile. Elle permet à la théorie de devenir plus importante que le monde lui-même, ce qui est inacceptable. Donc, ceux qui participent à l'élaboration et à l'évaluation de 
la politique gènérale en utilisant des structures conséquentialistes baséees sur la probabilité ne devraient pas rejeter le principe de reconsidération. Il est nécessaire parce qu'il garantit le lien vital entre la justification de la continuation de l'application d'une politique et l'avancement des buts que cette politique est destinee à faire avancer, lien auquel ceux d'entre nous qui se soucient du monde réel doivent réaffırmer leur soutien. Sans ce lien, nous risquons de devenir nos pires ennemis, faisant progresser activement des choses qu'au départ, nous essayions d'éviter.

Cet essai a été centré sur la politique de dissuasion, mais il a aussi posé des questions extrêmement importantes pour la politique générale. Le thème de la dissuasion nucléaire a récemment connu une grande popularité chez les philosophes. Ce qui est plus important, les problèmes concernant la politique de dissuasion nucléaire sont des problèmes inhérents à toute politique de dissuasion qui implique la possibilité d'utiliser la menace d'actions indésirables comme moyen de dissuasion. Ces problèmes vont probablement réapparaître à maintes reprises, surtout dans le domaine de la défense nationale. De plus, cet article a remis en question une certaine façon de faire de la philosophie, où la recherche de la cohérence logique se sépare tellement de toute situation réelle que la théorie nous joue des tours. En tant que théoriciens, nous devons y faire attention et surtout ne pas cesser de reconsiderer les politiques au fur et à mesure que l'avenir se présente à nous. Si l'on néglige de le faire, on refuse de voir le monde réel, ce qui arrive bien trop souvent en philosophie.

\section{Département de philosophie Université de Georgie}

